\title{
The Tibal Nerve (Posterior Tibal Nerve): Anatomical Course and Relation at the Ankle
}

\author{
Bilal Farhan and Gamal Ghoniem* \\ Department of Urology, University of California, USA
}

*Corresponding author: Dr. Gamal Ghoniem, MD, FACS, Professor\& Vice Chair of Urology, Chief Division of Female Urology, Pelvic Reconstruction Surgery \& Voiding Dysfunction, University of California, Irvine, 333 City Blvd. West, Ste. 2100, Orange, CA 92868. Email: farhanb@uci.edu

Submission: 眥 December 06, 2017; Published: 眥 February 23, 2018

\section{Introduction and Objective}

The Tibial nerve (TN), also known as posterior tibial nerve, the largest of the two major divisions of the sciatic nerve, is a continuation of the medial trunk of the sciatic nerve, which derives fibers from the ventral divisions of the L5, S1, and S3 roots, these nerves roots share same nerves roots to the detrusor, urinary sphincter, and pelvic floor muscles. Anatomical studies of the TN in the distal third of the leg and at the level ankle began to take more importance with the establishment and definition of course of the TN due to the wide clinical and research purposes of the TN and development of minimally invasive treatment of bladder dysfunction such as Percutaneous Tibial nerve stimulation (PTNS), nerve block and etc. [1,2]. We determined, through dissection of fresh cadaver, the neuroanatomy of the TN and its relation to the other structures at the ankle in relation to the tarsal tunnel.

\section{Dissection and Finding}

The position of the foot remained in neutral position and the surgical exploration was initiated $15 \mathrm{~cm}$ proximal to the center of the medial malleolus, continuing distally up to the medial surface of the foot. A longitudinal incision covered this entire distance and two transversal incisions, one at each end of the longitudinal incision, completed the approach, facilitating the elevation of two skin flaps, with sufficient exposure of the region The neurovascular bundle, which is wrapped in its own sheath, was then incised and the vascular structures separated from the $\mathrm{TN}$, thus exposing a significant part of the path of this nerve in front of, inside and behind the tarsal tunnel.

The TN near the ankle becomes superficial, lying on the medial side of the ankle, and then passing into the foot beneath the tarsal tunnel. The tarsal tunnel is a fibro-osseous tunnel located behind and below the medial malleolus. It has a bony floor, and the flexor retinaculum forms the roof. This is a thin, fan-shaped, fibrous tissue arising from the medioinferior aspect of the medial malleolus and inserting into the periosteum of the medial tuberosity of the calcaneus. The tunnel is narrowest at its distal aspect, where it blends into the fasciae of the abductor hallucis muscle. The tibial nerve is $3-4 \mathrm{~cm}$ from the medial malleolus (halfway between medial malleolus and the Achilles tendon) [3]. Besides the tibial nerve, the tendons of the posterior tibial, flexor digitorum longus, and the tibial artery and vein pass through this fibro-osseous channel (Figure 1\&2).

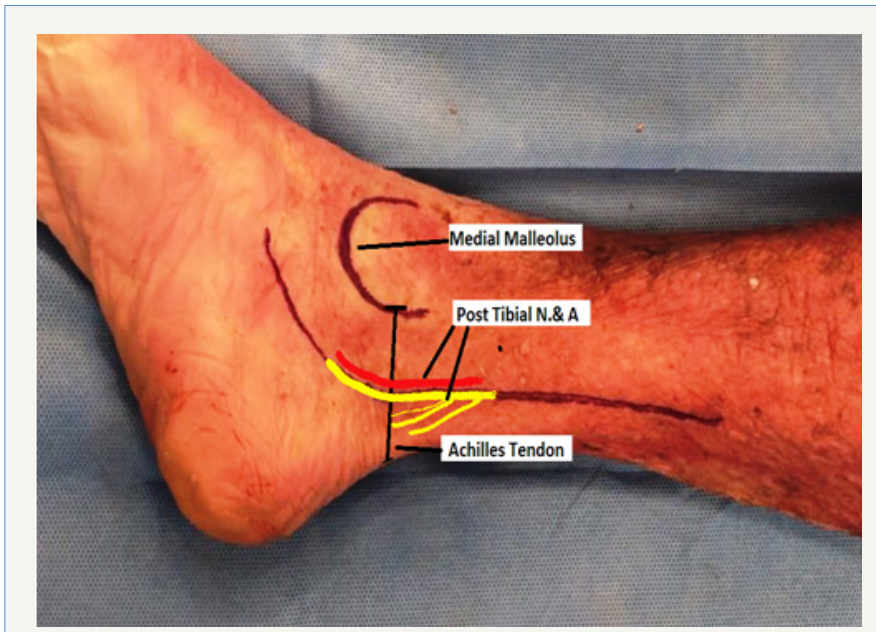

Figure 1: Surface anatomy of the medial aspect of the Ankle; showing the Posterior (Tibial nerve) mid-way between the medial malleolus and Achilles tendon.

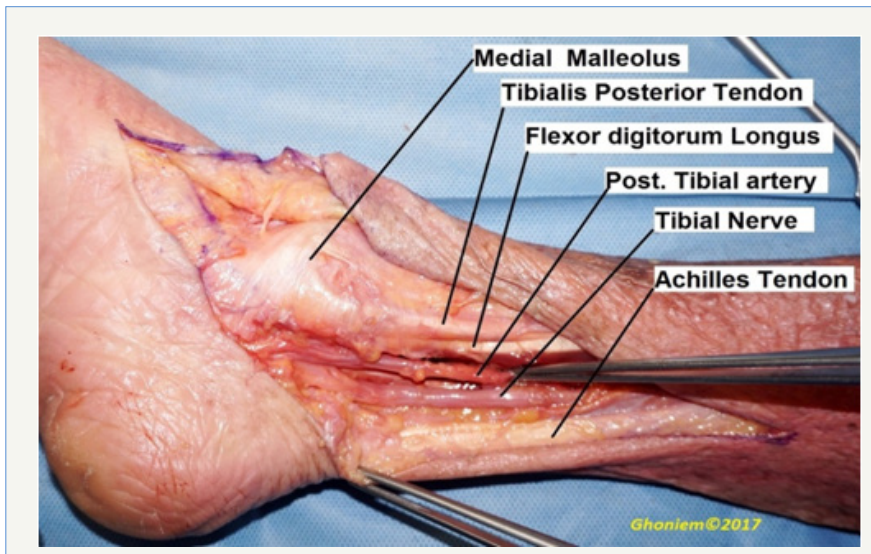

Figure 2: Relation of the Posterior (Tibial Nerve) in relation to the other structures on the medial aspect of the ankle. 


\section{Discussion}

The TN is one of the main branches of the sciatic nerve. It passes through the popliteal fossa and branches to posterior calf muscles. The TN becomes more superficial as it descends along the distal leg. As the bundle reaches the ankle region, it is positioned in a fibroosseous structure called the tarsal tunnel. This tunnel is bounded by the flexor retinaculum medially, the calcaneus and the talus laterally, and the medial malleolus anteriorly. Identification of the course of the TN and its branches on the medial aspect of the lower leg and around the tarsal tunnel is an important issue in various clinical

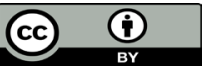

Creative Commons Attribution 4.0 International License

For possible submissions Click Here

\section{Submit Article}

fields including the PTNS uses in various bladder dysfunctions and chronic pelvic pain. This will help better understanding the surface anatomy and better result of its application.

\section{References}

1. Stoller ML (1999) Afferent nerve stimulation for pelvic floor dysfunction. Eur Urol 35(2): 16.

2. Myerson MS, Ruland CM, Allon SM (1992) Regional anesthesia for foot and ankle surgery. Foot Ankle 13(5): 282-288.

3. Farhan B, Ahmed A, Rahul D, Gamal G (2016) Percutaneous Tibial Nerve Stimulation in Urology: Overview. Women's Health \& Gynecology 2(5): $1-5$.

Your subsequent submission with Crimson Publishers will attain the below benefits

- High-level peer review and editorial services

- Freely accessible online immediately upon publication

- Authors retain the copyright to their work

- Licensing it under a Creative Commons license

- Visibility through different online platforms

- Global attainment for your research

- Article availability in different formats (Pdf, E-pub, Full Text)

- Endless customer service

- Reasonable Membership services

- Reprints availability upon request

- One step article tracking system 